Pacific Journal of Mathematics

A COMBINATORIAL PROBLEM; STABILITY AND ORDER
FOR MODELS AND THEORIES IN INFINITARY LANGUAGES 


\title{
A COMBINATORIAL PROBLEM; STABILITY AND ORDER FOR MODELS AND THEORIES IN INFINITARY LANGUAGES
}

\section{SAHARON SHELAH}

\begin{abstract}
Some infinite combinatorial problems of Erdös and Makkai are solved, and we use them to investigate the connection between unstability and the existence of ordered sets; we also prove the existence of indiscernible sets under suitable conditions.
\end{abstract}

o. Introduction. In $\S 1$ we deal with combinatorial problems raised by Erdös and Makkai in [5] (they appear later in Erdös and Hajnal [3], [18] Problem 71).

Let us define: $P 2(\lambda, \mu, \alpha)$ holds when for every set $A$ of cardinality $\mu$, and family $S$ of subsets of $A$ of cardinality $\lambda$, there are $a_{k} \in A, X_{k} \in S$ for $k<\alpha$, such that either $k, l<\alpha$ implies $a_{k} \in X_{l} \Leftrightarrow$ $k<l$ or $k, l<\alpha$ implies $a_{k} \in X_{l} \Leftrightarrow l \leqq k$.

Erdös and Makkai proved in [5] that if $\lambda>\mu \geqq \aleph_{0}$, then $P 2(\lambda$, $\mu, \omega)$ holds. Assuming G.C.H. for simlicity only, our theorems imply $P 2\left(\boldsymbol{\aleph}_{\beta+2}, \boldsymbol{\aleph}_{\beta+1}, \boldsymbol{\aleph}_{\beta}\right)$ holds for every $\beta$.

In $\S 2$ we mainly generalize results on stability from Morley [9] and Shelah [12] to models, and theories of infinitary languages. We first deal with stable models. Let $M$ be a model, $L$ the first-order

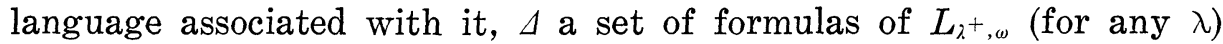
each with finite number of free variables. We shall assume $\Delta$ is closed under some simple operations. $M$ is $(\Delta, \lambda)$-stable, if for each $A \subset|M|,|A| \leqq \lambda$, the elements of $M$ realize over $A$ no more than $\lambda$ different $\Delta$-types. Let $\lambda \in \operatorname{Od}_{\Delta}(M)$ if there is $\varphi(\bar{x}, \bar{y}) \in \Delta$ and sequences $\bar{a}^{k}, k<\lambda$, of elements of $M$ such that for every $k, l<\lambda, M \vDash \varphi\left[\bar{a}^{k}, \bar{a}^{l}\right]$ if and only if $k<l$.

By Theorem 2.1, if $M$ is not $(\Delta, \kappa)$-stable $\kappa^{|\Delta|}=\kappa, \kappa=\sum_{\mu<\lambda}\left(\kappa^{\mu}+2^{2^{\mu}}\right)$, then $\lambda \in O d_{\Delta}(M)$. Theorem 2.2 says that if $M$ is $(\Delta, \lambda)$-stable, $\lambda \notin O d_{\Delta}(M)$, $\|M\|>\lambda, A \subset|M|,|A| \leqq \lambda$, and the cofinality of $\lambda$ is $>|\Delta|$, then in $M$ there is an indiscernible set over $A$ of cardinality $>\lambda$. This generalizes Theorem 4.6 of Morley [9] for models of totally transcendental theories.

A theory $T, T \subset L_{\lambda^{+},{ }_{\omega}}$ for some $\lambda$, is $(\Delta, \mu)$-stable, if every model of $T$ is $(\Delta, \mu)$-stable. By Theorem 2.4, if $T, \Delta \subset L_{\lambda^{+}, \omega}|T| \leqq \lambda$, and $\mu(\lambda) \in \operatorname{Od}_{\Delta}(M)$ for some model $M$ of $T$, then for every $\kappa, T$ is not $(\Delta, \kappa)$ stable. This is a converse of Theorem 2.1. (Morley [9] proved a particular case of this theorem (3.9) that if $T$ is a first-order, counta- 
ble, complete, totally trancendental theory, (i.e., $T$ is $\left(\Delta, \aleph_{0}\right)$-stable, where $\Delta$ is the set of all formulas of $L$ ), then $\aleph_{0} \notin O d_{\Delta}(M)$ for any model $M$ of $T$. (In fact he used a little stronger definition for $\left.\aleph_{0} \in O d_{\Delta}(M).\right)$

By Theorem 2.5, if $T \subset L_{\lambda^{+}, \omega}$, and $\Delta$ is arbitrary, and for every $\kappa, T$ is not $(\Delta, \kappa)$-stable, then for some $\Delta_{1} \subset L_{\lambda^{+}, \omega},\left|\Delta_{1}\right| \leqq \lambda, T$ is $\left(\Delta_{1}, \kappa\right)$ unstable for every $\kappa$. By Shelah [16], we deduce that for every $\kappa>|T|+\lambda, T$ has $2^{\kappa}$ nonisomorphic models of cardinality $\kappa$.

Notations. Let $\lambda, \kappa, \mu, \chi$ denote cardinals (infinite, if not clear otherwise). Let $\alpha, \beta, \gamma, i, j, k, l$ denote ordinals and $m, n$ denote natural numbers. We shall indentify cardinals with initial ordinals, and $\boldsymbol{\aleph}_{\alpha}$ will be the $\alpha$ th infinite cardinal ( $\boldsymbol{\aleph}_{0}$-the first). The first infinite ordinal is denoted by $\omega . \lambda^{+}$is the first cardinal greater than $\lambda$. $|A|$ is the cardinality of the set $A$.

1. Combinatorial problems. Let $A$ denote a set, $S$ a family of subsets of $A$. Let $A(-) S$ be the family $\{A-B: B \in S\} . A^{\alpha}$ is the set of sequences of length $\alpha$ of $A$; and if $\bar{a} \in A^{\alpha}, l(\bar{a})=\alpha$ and $\bar{a}_{\beta}$ is the $\beta$ th element in the sequence. After Erdös and Makkai [5], $\bar{a}$ if strongly cut by $S$ if for every $\beta<\alpha$, there is $X_{\beta} \in S$ such that $a_{\gamma} \in X_{\beta} \Leftrightarrow \gamma<\beta$ for every $\gamma, \beta<\alpha$. Erdös and Makkai [5] proved that is $|S|>|A| \geqq \boldsymbol{\aleph}_{0}$, then there is a sequence $\bar{a} \in A^{\omega}$ which is strongly cut by $S$ or by $A(-) S$. They asked several questions ([5] p. 159 and [3] problem 71 p. 45). We shall here answer some of their questions.

Let us define

DeFinition 1.1. $P 1(\lambda, \mu, \alpha)$ holds, if $|S|=\lambda,|A|=\mu$ implies there are $\bar{a}, \bar{b} \in A^{\alpha}, \bar{X} \in S^{\alpha}$ such that: for every $\beta, \gamma<\alpha$,

$$
\bar{a}_{\beta} \in \bar{X}_{\gamma} \Leftrightarrow \bar{b}_{\beta} \in \bar{X}_{\gamma} \text { if and only if } \gamma<\beta \text {. }
$$

Definition 1.2. $P 2(\lambda, \mu, \alpha)$ holds, if $|S|=\lambda,|A|=\mu$ implies there are $\bar{a} \in A^{\alpha}, \bar{X} \in S^{\alpha}$ such that:

$$
\text { either } \beta, \gamma<\alpha \text { implies } \bar{a}_{\beta} \in \bar{X}_{\gamma} \Longleftrightarrow \beta<\gamma
$$

or

$$
\beta, \gamma<\alpha \text { implies } \bar{a}_{\beta} \in \bar{X}_{\gamma} \Longleftarrow \gamma \leqq \beta \text {. }
$$

REMARK. This means that $\bar{a}$ is strongly cut by $S$ or by $A(-) S$.

Definition 1.3. $P 3(\lambda, \mu, \alpha)$ holds if $|S|=\lambda,|A|=\mu$ implies 
there are $\bar{a} \in A^{\alpha}, \bar{X} \in S^{\alpha}$ such that for every $\beta, \gamma<\alpha, \bar{a}_{\beta} \in \bar{X}_{\gamma} \Leftrightarrow \beta<\gamma$.

REMARK. This means $\bar{a}$ is strongly cut by $S$.

Notation. In each of $P 1, P 2, P 3$ we shall always implicitly assume $2^{\mu} \geqq \lambda>\mu$. For otherwise, those relations are not interesting.

Clearly, the theorem of [5] is by our notation, that $P 2\left(\lambda^{+}, \lambda, \omega\right)$ holds. Let us now list the results proved here about those three properties.

THEOREM 1.1. For every $\lambda, P 3\left(\lambda^{+}, \lambda, \omega\right)$ does not hold. (This solves negatively problem 1 in [5], which is the same as problem $71 \mathrm{~A}$, in [3] p. 45.) (In fact, we prove a stronger result.)

Theorem 1.2. If $\lambda>\sum_{0 \leqq \kappa<\chi}\left(\mu^{\kappa}+2^{2^{\kappa}}\right)$ then $P 1(\lambda, \mu, \chi)$ holds.

THEOREM 1.3. If $\lambda>\mu^{2^{\chi}}$ then $P 2\left(\lambda, \mu, \chi^{+}\right)$holds. Moreover if $\chi^{0}=\sum_{0 \leqq \kappa<\chi} 2^{\kappa}, \lambda>\mu^{\chi^{0}}$ then $P 2(\lambda, \mu, \chi)$ holds.

Theorem 1.4. If $P 1(\lambda, \mu, \chi)$ and $\chi \rightarrow(\kappa)_{4}^{2}$ holds, then $P 2(\lambda, \mu, \kappa)$ holds.

REmark. (1) $\chi \rightarrow(\kappa)_{4}^{2}$ is defined in Erdös, Hajnal and Rado [4]. As the proof is straightforward, we leave it to the reader.

(2) We can combine theorems 1.2 and 1.4 to get results about $P 2(\lambda, \mu, \alpha)$. For example by Ramsey [11], $\boldsymbol{\aleph}_{0} \rightarrow\left(\boldsymbol{\aleph}_{0}\right)_{4}^{2}$, hence $P 2(\lambda, \mu, \omega)$ holds (which is the result of [5]). (Here, as usual, we implicitly assume $\lambda>\mu \geqq \boldsymbol{\aleph}_{0}$.)

(3) Theorems 1.2, 1.3, 1.4 give partial answer to a question which naturally arises from [5], and problem 2, [5], and 71B [3] are the most simple cases of it.

THEOREM 1.5. P2( $\lambda, \mu, \omega+1)$ holds. Moreover, if $\lambda>\mu=\mu^{\aleph_{0}}$, $n<\omega$, then $P 2(\lambda, \mu, \omega+n)$ holds.

REMARK. This answers problem 3 of [5] (in fact even stronger) and partially answer problem 2 of [5] $(=71 \mathrm{~B}$ of [3]). The proof gives several more results of this kind.

To clarify our results let us assume G.C.H.

COROLlaRY 1.6. (G.C.H.) For every regular cardinality $\mu$, and any cardinal $\chi<\mu, P 2\left(\mu^{+}, \mu, \chi\right)$ holds. Moreover, if $\mu$ is singular, $\chi$ is less than the cofinality of $\mu$, then P2( $\left.\mu^{+}, \mu, \chi\right)$ holds. If $\chi$ is 
not greater than the cofinality of $\mu, P 1\left(\mu^{+}, \mu, \chi\right)$ holds.

Proof. Immediate from Theorems 1.2, 1.3, 1.4, and by [4], $\left(2^{\lambda}\right)^{+} \rightarrow$ $\left(\lambda^{+}\right)_{4}^{2}$ holds.

The question naturally arises whether those are the best possible results. Prikry essentially proved this. See [18] Problem. 72.

Theorem 1.7. Suppose $\lambda=\mu^{\chi}>\sum_{0 \leqq \kappa<\chi} \mu^{\kappa}=\mu_{0}$ then $P 2\left(\lambda, \mu_{0}, \chi+\right.$ 2) does not holds. $(\chi+2$-this is an ordinal addition). Moreover $P 1\left(\lambda, \mu_{0}, \chi+2\right)$ does not holds.

In [5], not $P 2\left(\boldsymbol{\aleph}_{1}, \boldsymbol{\aleph}_{0}, \omega+2\right)$ was proved; and as the proof is similar and straightforward we leave it to the reader.

The most simple open problems are: (for simplicity only we assume G.C.H.)

Problem 1. If $\boldsymbol{\aleph}_{\alpha}$ is regular, does $P 1\left(\boldsymbol{\aleph}_{\alpha+1}, \boldsymbol{\aleph}_{\alpha}, \boldsymbol{\aleph}_{\alpha}\right)$ hold? Does $P 2\left(\aleph_{\alpha+1}, \aleph_{\alpha}, \aleph_{\alpha}\right)$ hold?

Problem 2. If $\boldsymbol{\aleph}_{\alpha}$ singular, $\boldsymbol{\aleph}_{\beta}$ is the cofinality of $\boldsymbol{\aleph}_{\alpha}$, does $P 2\left(\boldsymbol{\aleph}_{\alpha+1}, \boldsymbol{\aleph}_{\alpha}, \boldsymbol{\aleph}_{\beta}\right)$ hold?

Maybe the answers are independent of $Z F+A C$.

Let us summarize the trivial facts about our properties.

Lemma 1.8. (A) If $\lambda_{1} \geqq \lambda, \mu_{1} \leqq \mu, \alpha_{1} \leqq \alpha$ and $P 1(\lambda, \mu, \alpha)$ hold, then $P 1\left(\lambda_{1}, \mu_{1}, \alpha_{1}\right)$ holds. The same is ture for $P 2$ and $P 3$.

(B) $P 3(\lambda, \mu, \alpha)$ implies $P 2(\lambda, \mu, \alpha) ; P 2(\lambda, \mu, \alpha)$ implies $P 1(\lambda, \mu, \alpha)$, where $\alpha$ is a limit ordinal; and $P 2(\lambda, \mu, \alpha+1)$ implies $P 1(\lambda, \mu, \alpha)$.

(C) If $\alpha<\omega, \lambda>\mu$ then $P 3(\lambda, \mu, \alpha)$ holds.

(D) If cf $(\lambda) \leqq \mu<\lambda,(\forall \chi<\lambda) \neg P 2(\chi, \mu, \alpha)$ then not $P 2(\lambda, \mu, \alpha)$.

Proof. Immediate. We use $(D)$ for $(B)$.

Let us now prove the theorems.

Definition 1.4. Ded $(\mu)$ is the first cardinal $\lambda$ such that there is no ordered set of cardinality $\lambda$ with a dense subset of cardinality $\mu$.

Remark. Clearly $\mu^{+}<\operatorname{Ded}(\mu) \leqq\left(2^{\mu}\right)^{+}$. By Mitchell [8] it is consistent with $Z F+A C$ that $\operatorname{Ded}\left(\mathbf{\aleph}_{1}\right)<\left(2^{\aleph_{1}}\right)^{+}$.

Theorem 1.9. If $\mu<\lambda<\operatorname{Ded}(\mu)$ then $P 3(\lambda, \mu, \omega)$ does not hold.

REMARK. Clearly Theorem 1.1 is an immediate conclusion of this theorem. 
Proof. Let a tree mean a pair of a set and a well ordering of the set, which is not necessarily a total ordering. A branch of a tree is a maximal ordered subset. It can be easily shown that there is a tree $\langle A,\langle\rangle(A$-the set, $<$-the ordering) such that $|A|=\mu$ and the tree has $\geqq \lambda$ branches. Let $S_{1}$ be the family of the branches of the tree and $S=A(-) S_{1}$. Clearly $|S| \geqq \lambda,|A|=\mu$ and $S$ is a family of subsets of $A$. So it suffices to show that there is no $\bar{a} \in A^{\omega}$ which is strongly cut by $S$.

So suppose $\bar{a} \in A^{\omega}$ is strongly cut by $S$. By using Ramsey theorem ([11]) we know there is an infinite subsequence of $\bar{a}, \bar{b}$, such that exactly one of the following conditions is fulfilled

(1) for every $n<m<\omega, \bar{b}_{n}<\bar{b}_{m}$ (in the tree)

(2) for every $n<m<\omega, \bar{b}_{n}=\bar{b}_{m}$

(3) for every $n<m<\omega, \bar{b}_{n}>\bar{b}_{m}$

(4) for every $n<m<\omega, b_{n} b_{m}$ are incomparable, i.e., $b_{n} \neq b_{m}$, not $b_{n}>b_{m}$, and not $b_{n}<b_{m}$.

Now clearly also $\bar{b}$ is strongly cut by $S$. Hence (2) cannot be fulfilled. As $<$ is a well ordering (3) cannot be fulfilled. Now as $\bar{b}$ is strongly cut by $S$, there is a branch of $\langle A,\langle\rangle$ which contains two of the $b_{n}$ 's and so they are comparable, in contradiction to (4). So (1) is fulfilled. As $\bar{b}$ is strongly cut by $S$, there is $X \in S$ such that $\bar{b}_{0} \in X, \bar{b}_{1} \notin X$. But $A-X$ is a branch of the tree, $\bar{b}_{1} \in A-X$, $\bar{b}_{0}<\bar{b}_{1}$, hence $\bar{b}_{1} \in A-X$, a contradiction.

\section{THEOREM 1.2. If $\lambda>\sum_{0 \leqq \kappa<\chi}\left(\mu^{\kappa}+2^{2 \kappa}\right)$ then $P 1(\lambda, \mu, \chi)$ holds.}

Proof. Let $S$ be a family of subsets of $A,|S|=\lambda,|A|=\mu$. We should prove there are $\bar{a}, \bar{b} \in A^{*}$ and $\bar{X} \in S^{*}$ such that, for every $\alpha, \beta<\chi, \bar{a}_{\alpha} \in \bar{X}_{\beta} \Leftrightarrow \bar{b}_{\alpha} \in \bar{X}_{\beta}$ iff $\beta<\alpha$.

Let us define, for every $T \subset S$, an equivalence relation $E_{T}$ on $A: a E_{T}$ $b$ holds if and only if for every $X \in T, a \in X \Leftrightarrow b \in X$. Clearly $E_{T}$ is an equivalence relation, and the number of equivalence classes is $\leqq 2^{|T|}$.

Let us also define that $T \subset S$ fixes $X \in S$ if for every $a, b \in A$, $a E_{T} b$ implies $a \in X \Leftrightarrow b \in X$. Clearly the number of $X \in S$ which are fixed by $T$ cannot be more than the number of subsets of the set of the $E_{T}$-equivalence classes. Hence $\mid\{X: X \in S, X$ is fixed by $T\} \mid \leqq 2^{2|T|}$.

Let us now define by induction the families $S_{\kappa}$ for $0 \leqq \kappa<\chi$ such that:

(1) $S_{\kappa} \subset S,\left|S_{\kappa}\right| \leqq \mu^{k}$

(2) $\kappa_{1}<\kappa_{2}$ implies $S_{\kappa_{1}} \subset S_{\kappa_{2}}$

(3) if $B, C \subset A,|B| \leqq \kappa,|C| \leqq \kappa$, and there is $X \in S$ such that $B \subset X, C \cap X=0$, then there is $Y \in S_{\kappa}$ such that $B \subset Y, C \cap Y=0$.

Clearly we can define the $S_{\kappa}$. We shall now prove that 
(*) there is $Y \in S$ such that for any $T, T \subset S_{\kappa}, 0 \leqq \kappa<\chi,|T| \leqq$ $\kappa, Y$ is not fixed by $T$.

Suppose $(*)$ does not hold and we shall get a contradiction. So

$$
S=\bigcup_{0 \leqq \kappa<x} \bigcup_{\substack{T \\|T| \leqq \kappa}}\{X: X \in S, X \text { is fixed by } T\} \text {. }
$$

We have proved that $\mid\{X: X \in S, X$ is fixed by $T\} \mid \leqq 2^{2|T|}$, and by its contruction $\left|S_{\kappa}\right| \leqq \mu^{\kappa}$. Hence

$$
\begin{aligned}
\lambda=|S| & \leqq \sum_{0 \leqq n<\chi} \sum_{\substack{T<S_{\kappa} \\
|T| \leqq \kappa}} 2^{2|T|} \\
& \leqq \sum_{0 \leqq \kappa<\chi}\left|S_{\kappa}\right|^{\kappa} \times 2^{2^{\kappa}}=\sum_{0 \leqq \kappa<\chi}\left(\left|S_{\kappa}\right|^{\kappa}+2^{2 \kappa}\right) \\
& \leqq \sum_{0 \leqq \kappa<\chi}\left(\mu^{\kappa}+2^{2 \kappa}\right)<\lambda
\end{aligned}
$$

a contradiction. So $(*)$ holds.

Now we shall define by induction $a_{k}, b_{k}, X_{k}$ for $k<\chi$ such that:

(A) $a_{k} \in A, b_{k} \in A$, and $X_{k} \in S_{|k|+1}$

(B) if $l \leqq k$ then $a_{l} \in X_{k}, a_{l} \in Y, b_{l} \notin X_{k}$, and $b_{l} \notin Y$

(C) if $l<k$, then $a_{k} \in X_{l}$ if and only if $b_{k} \in X_{l}$.

Suppose $a_{l}, b_{l}$ and $X_{l}$ has been defined for every $l<k$. Let $1+|k|=\kappa$, and $T=\left\{X_{l}: l<k\right\}$. Clearly $T \subset S_{\kappa},|T| \leqq \kappa$. Hence, by the definition of $Y$, it is not fixed by $T$. So there are $a_{k}, b_{k} \in A$ such that: $a_{k} \in Y, b_{k} \notin Y$ and $a_{k} E_{t} b_{k}$, i.e., for every $l<k, a_{k} \in X_{l}$ if and only if $b_{k} \in X_{l}$. Clearly $\left\{a_{l}: l \leqq k\right\} \subset Y,\left\{c_{l}: l \leqq k\right\} \cap Y=0,\left|\left\{a_{l}: l \leqq k\right\}\right| \leqq$ $\kappa,\left|\left\{b_{l}: l \leqq k\right\}\right| \leqq \kappa$; hence by the definition of $S_{\kappa}$ there is $X_{k} \in S_{\kappa}$ such that

$$
\left\{a_{l}: l \leqq k\right\} \subset X_{k},\left\{b_{l}: l \leqq k\right\} \cap X_{k}=0 .
$$

Clearly $\left\langle a_{k}: k\langle\chi\rangle,\left\langle b_{k}: k\langle\chi\rangle\right.\right.$, and $\left\langle X_{k}: k\langle\chi\rangle\right.$ are the required sequences, and so Theorem 1.2 is proved.

THEOREM 1.3. If $\chi^{0}=\sum_{0 \leqq \kappa<\chi} 2^{\kappa}, \lambda>\mu^{\chi^{0}}$, then $P 2(\lambda, \mu, \chi)$ holds.

Proof. As the proof is very similar to the proof of Theorem 2, we shall only sketch it.

Suppose $S$ is a family of subsets of $A,|S|=\lambda,|A|=\mu$. It is easy to find $S_{1} \subset S,\left|S_{1}\right| \leqq \mu^{\chi^{0}}$ such that:

(1) if $B \subset A,|B| \leqq 2^{\kappa}, 0 \leqq \kappa<\chi$, and $T \subset S_{1},|T| \leqq \kappa$ and $Y \in S$ then there is $X \in S_{1}$ such that: (A) $X \cap B=Y \cap B$ (B) if $C$ is an $E_{T}$-equivalence class then $C \subset X \Leftrightarrow C \subset Y$ and $C \cap X=0 \Leftrightarrow C \cap Y=0$.

(2) if $X_{l}^{k}, k<\alpha_{l}<\chi, l<\chi^{0}, Y_{l}^{k}, k<\beta_{l}<\chi, l<\chi^{0}$ and $Z_{l}, l<\chi^{0}$ are sets from $S_{1}$, and there is $X \in S$ such that: for every $l<\chi^{0}$ 


$$
X \cap \bigcap_{k<\alpha_{l}} X_{l}^{k} \cap \bigcap_{k<\beta_{l}}\left(A-Y_{l}^{k}\right)=Z_{l} \cap \bigcap_{k<\alpha_{l}} X_{l}^{k} \cap \bigcap_{k<\beta_{l}}\left(A-Y_{l}^{k}\right)
$$

then there is $X \in S_{1}$, which satisfies this condition.

Now we can repeat a construction similar to that which appears in the proof of Theorem 1.

As Theorem 1.4 is trivial, it remains to prove only

THEOREM 1.5. (A) If $\lambda>\mu$ then $P 2(\lambda, \mu, \omega+1)$ holds.

(B) If $\lambda>\mu=\sum_{0 \leqq r<\chi} \mu^{\kappa}, \alpha \leqq \chi$ and $P 2(\lambda, \mu, \alpha)$ holds then $P 2(\lambda, \mu, \alpha+1)$ holds. Hence for every $n$, if in addition $\alpha<\chi$, $P 2(\lambda, \mu, \alpha+n)$ holds. (By $1.8 \mathrm{D}$ we can assume $\operatorname{cf}(\lambda)>\mu)$.

(C) If $\lambda>\mu^{\aleph_{0}}$, then $P 2(\lambda, \mu, \omega+n)$.

Remark. (1) Clearly (A) cannot be improved by [5] $P 2\left(\boldsymbol{\aleph}_{1}\right.$, $\left.\aleph_{0}, \omega+2\right)$ does not hold.

(2) Part of the proof is a generalization of a proof of A. Máté which appeared in [5].

Proof. As the proof of (B) is obvious from the proof of $A$, we shall prove $A$ only. (C follow from $\mathrm{B}$ ).

So let $S$ be a family of subsets of $A,|S|=\lambda,|A|=\mu$.

First, there is $a^{0} \in A$ such that $S_{1}=\left\{X: X \in S, a^{0} \in X\right\}$ is of cardinality $>\mu$. Otherwise

$$
\begin{aligned}
\lambda=|S| & =\left|\bigcup_{a \in A}\{X: X \in S, a \in X\} \cup\{0\}\right| \\
& \leqq \sum_{a \in A}|\{X: X \in S, a \in X\}|+1=\mu \cdot \mu+1=\mu<\lambda
\end{aligned}
$$

a contradiction. Similarly there is $\alpha^{1} \in A$ such that $S_{2}=\left\{X: X \in S_{1}\right.$, $\left.a^{1} \notin X\right\}$ is of cardinality $>\mu$. Now at first we assume

(*) there is $A^{1} \subset A$, and $S^{1} \subset\left\{Y \cap A^{1}: Y \in S_{2}\right\}$ such that $\left|S^{1}\right|>\mu$; and for every $X \in S^{1}$,

$$
\left|\left\{Y \cap X: Y \in S^{1}\right\}\right| \leqq \mu
$$

Then it can be easily seen that if $X_{1}, \cdots, X_{n} \in S^{1}, X=X_{1} \cup \cdots \cup X_{n}$ then

$$
\left|\left\{Y \cap X: Y \in S^{1}\right\}\right| \leqq \mu \text {. }
$$

So we can easily find $S^{2} \subset S^{1},\left|S^{2}\right| \leqq \mu$ such that: if $X_{1}, \cdots, X_{n} \in S^{2}$, $X \in S^{1}$ and $X \subset X_{1} \cup \cdots \cup X_{n}$ then $X \in S^{2}$; and if $a_{0}, \cdots, a_{n} \in A, X \in S^{1}$, then there is $Y \in S^{2}$ such that $\left\{a_{0}, \cdots, a_{n}\right\} \cap X=\left\{a_{0}, \cdots, a_{n}\right\} \cap Y$. Now let $Y^{0} \in S^{1}, Y^{0} \notin S^{2}$. ( $Y^{0}$ exists as $\left.\left|S^{1}\right|>\mu \geqq\left|S^{2}\right|\right)$. Now we shall define by induction on $n, a_{n}, X_{n}$ such that: $a_{n} \in Y^{0}, X_{n} \in S^{2}$, and 
$a_{n} \notin X_{0}, a_{n} \notin X_{1}, \cdots, a_{n} \notin X_{n} ; a_{0}, \cdots, a_{n-1} \in X_{n}$. Suppose $a_{n}, X_{n}$ has been defined for every $n<m<\omega$. As $Y^{0} \notin S^{2}, Y^{0} \not \subset X_{0} \cup \cdots \cup X^{m-1}$, hence there is $a_{m} \in Y^{0}, a_{m} \notin X_{0} \cup \cdots \cup X^{m-1}$. Also there is $X_{m} \in S^{2}$ such that $\left\{a_{0}, \cdots, a_{m}\right\} \cap X_{m}=\left\{a_{0}, \cdots, a_{m}\right\} \cap Y^{0}$.

Now clearly if we define $a_{\omega}=\alpha^{1}$, clearly $\left\langle a_{\alpha} \mid \alpha<\omega+1\right\rangle \in A^{\omega+1}$ and is strongly cut by $S$; so the conclusion of theorem holds.

Similarly the conclusion of the theorem holds if

(**) there is $A^{1} \subset A$ and $S^{1} \subset\left\{Y \cap A^{1}: Y \in S_{2}\right\}$ such that $\left|S^{1}\right|>\mu$, and for every $X \in S^{1}$

$$
\left|\left\{Y \cap\left(A^{1}-X\right): Y \in S^{1}\right\}\right| \leqq \mu .
$$

Hence we can assume $(*)$ and $(* *)$ do not hold. So there is $X^{0} \in S_{2}$ such that $S_{3}=\left\{Y \cap X^{0}: Y \in S_{2}\right\}$ is of cardinality $>\mu_{\text {. }}$ (Otherwise, taking $A^{1}=A, S^{1}=S_{2},(*)$ holds.) Similarly there is $X^{1} \in S_{3}$ such that $S_{4}=\left\{Y \cap\left(X^{0}-X^{1}\right): Y \in S_{3}\right\}$ is of cardinality $>\mu$ (otherwise taking $A^{1}=X^{0}, S^{1}=S_{3},(* *)$ holds). Now $\left|S_{4}\right|>\mu \geqq\left|X^{0}-X^{1}\right|$, and $S_{4}$ is a family of subsets of $X^{0}-X^{1}$. Hence there is $\bar{a} \in\left(X^{0}-\right.$ $\left.X^{1}\right)^{\omega}$ which is strongly cut by $S_{4}$ or by $\left(X^{0}-X^{1}\right)(-) S_{4}$. Taking as $\bar{a}_{\omega}, a^{0}$ or $a^{1}$ (accordingly), we get a sequence from $A^{\omega+1}$ which is strongly cut by $S$ or $A(-) S$. So we prove Thorem 1.5A.

Naturally the question arises on the finite case. More exactly

DeFinition 1.5. For natural numbers $m, n$ let $f(m, n)$ be the first ordinal $\alpha$ such that $P 3(\alpha, m, n)$ holds.

The result is $f(m, n)=1+\sum_{k=0}^{n-1}\left(\begin{array}{c}m \\ k\end{array}\right)$. The proof follows from a little more complex result, of Perles and Shelah.

Another natural generalization is the relation $P 4(\lambda, \mu, \chi)$ which is

Definition 1.5. $P 4(\lambda, \mu, \chi)$ holds if whenever $|S|=\lambda,|A|=\mu$, and $S$ is a family of subsets of $A$, there exists $B \subset A,|B|=\chi$, such that for every $C \subset B$ there is $X \in S$ such that $X \cap B=C$.

Clearly $P 4(\lambda, \mu, \chi)$ implies $P 3(\lambda, \mu, \chi)$ and $P 3(\lambda, \mu, \alpha)$ for every $\alpha<\chi^{+}$. The only result known to me is that if $\lambda \geqq \operatorname{Ded}(\mu), \lambda$ is regular and $\chi$ is finite, then $P_{4}(\lambda, \mu, \chi)$ holds. (see Shelah [15]). Perles and I prove that if $\mu$ and $\chi$ are finite $P 4(\lambda, \mu, \chi)$ holds if and only if $\lambda>\sum_{k=0}^{\chi-1}\left(\begin{array}{l}\mu \\ k\end{array}\right)$. Later and independently Sauer [19] proved it.

2. On stable models and theories. In this section we shall apply a combinatorial theorem from $\S 1$ to get results in the theory of models.

Let $L$ be a first-order language; $L_{\lambda, \omega}$ will be its extension by permitting conjunctions on sets of $<\lambda$ formulas, provided that in the conjunction, only finitely many variables appear free. $L_{\infty, \omega}$ will be 
the class of formulas $\bigcup_{\lambda} L_{\lambda, \omega}$. $T$ will denote a set of sentences from $L_{\infty, \omega} \cdot \Delta$ will denote a set of formulas $\varphi(\bar{x})$ from $L_{\infty, \omega}$ (more exactly, $\Delta$ is a set of pairs $\langle\varphi, \bar{x}\rangle$ where $\varphi \in L_{\infty, \omega}, \bar{x}$ is a finite sequence of variables, and every free variable of $\varphi$ appears in $\bar{x}) . \quad \Delta$ is closed if it is closed under negation, finite conjunction (hence all connective), adding dummy variables and changing the order of the variables. $\bar{J}$ is the closure of $\Delta . M, N$ shall denote models ( $L$-models, if not said otherwise). $|M|$ is the set of elements of $M$. If $A \subset|M|, p$ is a $(\Delta, m)$-type over $A$ iff $p$ is a set whose elements are of the form $\varphi(\bar{x}, \bar{a})$ where $\bar{x}=\left\langle x_{0}, \cdots, x_{m-1}\right\rangle, \varphi(\bar{x}, \bar{y}) \in \Delta$ and $\bar{a} \in A$ (or more exactly $\left.\bar{a}_{0}, \bar{a}_{1}, \cdots \in A\right)$.

For $\bar{c} \in|M|$, the $\Delta$-type $\bar{c}$ realizes over $A, p(\bar{c}, A, M, \Delta)$ is

$$
\{\varphi(\bar{x}, \bar{a}): \bar{a} \in A, \varphi(\bar{x}, \bar{y}) \in \Delta, M \vDash \varphi[\bar{c}, \bar{a}]\} .
$$

Let

$$
S^{m}(A, M, \Delta)=\left\{p(\bar{c}, A, M, \Delta): \bar{c} \in|M|^{m}\right\} \text {. }
$$

The model $M$ is called $(\Delta, \lambda)$-stable if $|A| \leqq \lambda$ implies $\left|S^{1}(A, M, \Delta)\right| \leqq$ $\lambda$; otherwise $M$ is $(\lambda, \Delta)$-unstable.

Let $\lambda \in \operatorname{Od}_{\Delta}(M)$ if there is $n<\omega$, and sequences $\bar{a}^{l} \in|M|^{n}, l<\lambda$; and a formula $\varphi(\bar{x}, \bar{y}) \in \Delta$ such that $M \vDash \varphi\left[\bar{a}^{k}, \bar{a}^{l}\right]$ if and only if $k<l$ for every $k, l<\lambda$.

Theorem 2.1. Suppose $M$ is $(\Delta, \kappa)$-unstable, $\Delta=\bar{\Delta}, \kappa=\sum_{0 \leqq \mu<\lambda}\left(\kappa^{\mu}+\right.$ $2^{2^{\mu}}$ ) and $\kappa=\kappa^{|\Delta|}$. Then $\lambda \in \operatorname{Od}^{\Delta}(M)$.

Proof. Let $\Delta=\left\{\varphi_{k}\left(x, \bar{y}^{k}\right): k<|\Delta|\right\}, \Delta_{k}=\left\{\varphi_{k}\left(x, \bar{y}^{k}\right)\right\}$. As $M$ is $(\Delta$, $\kappa)$-unstable, there is $A \subset|M|,|A| \leqq \kappa$ such that $\left|S^{1}(A, M, \Delta)\right|>\kappa$. If for every $k<|\Delta|,\left|S^{1}\left(A, M, \Delta_{k}\right)\right| \leqq \kappa$ then

$$
\kappa<\left|S^{1}(A, M, \Delta)\right| \leqq\left|\prod_{k<|\Delta|} S^{1}\left(A, M, \Delta_{k}\right)\right|=\prod_{k<|\Delta|}\left|S^{1}\left(A, M, \Delta_{k}\right)\right| \leqq \kappa^{|\Delta|}=\kappa
$$

a contradiction. Hence there is $k<\kappa$ such that $\left|S^{1}\left(A, M, \Delta_{k}\right)\right|>\kappa$. Let $\varphi=\varphi_{k}$. Now clearly $S^{1}\left(A, M, \Delta_{k}\right)$ is a set of subsets of

$$
\Phi=\left\{\varphi_{k}(x, \bar{a}): \bar{a} \in A, \bar{a} \text { is of the length of } \bar{y}^{k}\right\} \text {. }
$$

Clearly $|\Phi| \leqq \kappa$. Hence by Theorem 1.2, there are $p_{l} \in S^{1}\left(A, M, \Delta_{k}\right)$ $\bar{a}^{l}, \bar{b}^{l} \in|A|$ for $l<\lambda$ such that $\varphi\left(x, \bar{a}^{l}\right) \in p_{j} \Leftrightarrow \varphi\left(x, \bar{b}^{l}\right) \in p_{j}$ if and only if $j<l$. Let $p_{l}=p\left(\bar{c}^{l}, A, M, \Delta_{k}\right.$ ), and $\bar{d}^{l}=\bar{a}^{l} \frown \bar{b}^{l} \frown \bar{c}^{l}$ (the juxtaposition of the three sequences). Clearly $M \vDash \varphi\left[\bar{c}^{j}, \bar{a}^{l}\right] \equiv \varphi\left[\bar{c}^{j}, \bar{b}^{l}\right]$ if and only if $j<l$. As $\Delta=\bar{\Delta}$, we can easily find $\psi(\bar{x}, \bar{y}) \in \Delta$ such that for $k, l<\lambda ; M \vDash \psi\left[\bar{d}^{k}, \bar{d}^{l}\right]$ if and only if $k<l$. Hence $\lambda \in \operatorname{Od}_{4}(M)$. 
Definition 2.1. Let $A, C \subset|M| . \quad C$ is $\Delta$-indiscernible over $A$ in $M$ if for every $n$, and every $n$ different elements $c_{0}, \cdots, c_{n-1}$ of $C$, and every additional $n$ different elements $c^{0}, \cdots, c^{n-1}$ of $C$

$$
p\left(\left\langle c_{0}, \cdots, c_{n-1}\right\rangle, A, M, \Delta\right)=p\left(\left\langle c^{0}, \cdots, c^{n-1}\right\rangle, A, M, \Delta\right) \text {. }
$$

Theorem 2.2. Suppose $M$ is $(\bar{d}, \lambda)$-stable, $\lambda \notin O d_{\bar{A}}(M), A \subset|M|$, $C \subset|M|,|A| \leqq \lambda<|C|$, and the cofinality of $\lambda$ is greater than $|\Delta|$. Then there exists $C_{1} \subset C,\left|C_{1}\right|>\lambda$ such that $C_{1}$ is 4 -indiscernible in $M$ over $A$.

REMARK. Taking a Souslin tree, we can see that the condition $\lambda \notin \operatorname{Od}_{\bar{A}}(M)$ is necessary. (More exactly, this is consistent with $Z F+$ AC.) Instead $c f(\lambda)>|\Delta|$ we can demand $\exists \mu<\lambda, \mu \notin O d_{\bar{\Delta}}(M)$.

Morley in [9] Theorem 4.6 proved a similar theorem for models of a complete, first-order, countable, totally transcendental theory. In [12] this was generalized to models of stable theories, and in [13], Theorem 3.1 to models with stable finite diagram. Another generalization is Theorem 5.9A of Shelah [15]. Theorem 2.2, in fact, implies all these theorems. (For 5.9A [15] we should note that if $\Delta$ is finite, then there is a finite $\Delta_{1}, \Delta \subset \Delta_{1} \subset \bar{\Delta}$, such that for any $M, \lambda ; M$ is $\left(\Delta_{1}, \lambda\right)$ stable if and only if it is $(\bar{\Delta}, \lambda)$-stable.)

Proof. As the proof is very similar to the proof of Theorem 3.1 [13], we omit it.

Definition 2.2. $T$ is $(\Delta, \lambda)$-stable if every model of $T$ is $(\Delta, \lambda)$ stable. $T$ is $\Delta$-stable, if for at least one $\lambda$ it is $(\Delta, \lambda)$-stable, $T$ is $(\Delta, \lambda)$-unstable [ $\Delta$-unstable] if it is not $(\Delta, \lambda)$-stable [ $\Delta-$-stable]. Let $\lambda \in O d_{\Delta}(T)$ if for at least one model $M$ of $T, \lambda \in O d_{\Delta}(M) . T$ is stable if it is $\Delta$-stable for every $\Delta$; otherwise-unstable.

REMARK. If $T$ has no model of cardinality $>\lambda$, then it is $(\Delta, \lambda)$ stable, and hence stable.

Theorem 2.3. Suppose $T, \Delta \subset L_{\lambda^{+},{ }_{\omega}},|T| \leqq \lambda,|L| \leqq \lambda, T$ is $(\Delta, \kappa)-$ unstable, $\kappa^{\mu(\lambda)}=\kappa$. Then $T$ is $\Delta$-unstable.

REMARK. (1) $\mu(\lambda)$ is the first cardinality such that if a sentence of a language $L_{i^{+}, \omega}^{1}$ has a model of cardinality $\mu(\lambda)$, it has models in any cardinalty $\geqq \lambda$.

(2) We can demand only: $T, \Delta \subset L_{\lambda^{+}, \omega},|T|+|\Delta| \leqq \lambda$, and for every $\mu<\mu(\lambda)$ there is $\kappa=\kappa^{\mu}$ such that $T$ is $(\Delta, \kappa)$-unstable.

(3) We can demand only $T, \Delta \subset L_{\lambda^{+}, \omega},|T| \leqq \lambda,|L|<\mu(\lambda), \kappa=$ 
$\sum_{\mu<\mu(\lambda)} \kappa^{\prime \prime}$ and $T$ is $(\Delta, \kappa)$-unstable.

Proof. Here we use Ehrefeucht-Mostowski models (see [2]) and the method of Morley [10]. All the results we use appeared in Chang [1]. As $T$ is $(\Delta, \kappa)$-unstable, $T$ has a model $M$ and $A \subset|M|$ such that $\left|S^{1}(A, M, \Delta)\right|>\kappa \geqq|A|$. It is well known that $\chi<\mu(\lambda)$ implies $2^{x}<\mu(\lambda)$; hence $\chi<\mu(\lambda)$ implies $2^{2 \chi}<\mu(\lambda)$. So $\kappa=\sum_{x<\mu(\lambda)}\left(\kappa^{\chi}+2^{2^{\chi}}\right)$. As $|\Delta| \leqq\left|L_{\lambda^{+}, \omega}\right|<\mu(\lambda)$, exactly as in the proof of Theorem 2.1, this implies that there are sequences $\bar{a}^{k}, \bar{b}^{k}, k<\mu(\lambda)$ from $A$ and $c_{k} \in|M|$, $k<\mu(\lambda)$ and a formula $\varphi(x, \bar{y}) \in \Delta$ such that:

for every $k, l<\mu(\lambda), M \vDash \varphi\left[c_{l}, \bar{a}^{k}\right] \equiv \varphi\left[c_{l}, \bar{b}^{k}\right] \quad$ if and only if $l<k$.

Now we add to $M$ the one place relation $P^{M}=\left\{c_{k}: k<\mu(\chi)\right\}$, and the functions $F_{1}^{M}, F_{2}^{M}$ defined by $F_{1}^{M}\left(\bar{a}^{k}\right)=c_{k}, F_{2}^{M}\left(\bar{b}^{k}\right)=c^{k}$, and otherwise $F_{1}^{M}(\bar{a}) \notin P^{M}, F_{2}^{M} \notin P^{M}$.

Now using Morley's method we get (in fact we need an improvement of Chang [1]):

(*) for every ordered set $I$, there is a model $M_{I}$ of $T$, in which there are $c_{s}, \bar{a}_{s}, \bar{b}_{s}$ for every $s \in I$ such that: for every $s, t \in I$

$$
M_{I} \vDash \varphi\left[c_{t}, \bar{a}_{s}\right] \equiv\left[c_{t}, \bar{b}_{s}\right] \text { if and only if } t<s .
$$

Let $\chi$ be any cardinality, and we shall prove $T$ is $(\Delta, \chi)$-unstable. We can find easily an ordered set $I,|I|>\chi$, with a dense subset $J$, $|J| \leqq \chi \quad$ (If $\chi_{1}=\inf \left\{\chi_{1}: 2^{\chi_{1}}>\chi\right\}$, then $I$ can be the set of sequences of ones and zeroes of length $\chi_{1}$, ordered lexicographically.) Let $M=M_{I}$, and let $A=\bigcup\left\{\right.$ Rang $\bar{a}_{s} \cup$ Rang $\left.\bar{b}_{s}: s \in J\right\}$. Clearly $|A| \leqq \boldsymbol{\aleph}_{0}+|J| \leqq$ $\chi$. On the other hand we shall show that $t_{1} \neq t_{2}, t_{1}, t_{2} \in I$ implies $p\left(c_{t_{1}}, A, M, \Delta\right) \neq p\left(c_{t_{2}}, A, M, \Delta\right)$. Hence $\left|S^{1}(A, M, \Delta)\right|>\chi$, so $T$ is $(\Delta, \chi)$ unstable.

Suppose $t_{1} \neq t_{2}, t_{1}, t_{2} \in I$. Without loss of generality suppose $t_{1}<$ $t_{2}$. As $J$ is a dense subset of $I$, there is $s \in J, t_{1}<s<t_{2}$. By the definition of $M_{I}$,

$$
\begin{gathered}
M \vDash \varphi\left[c_{t_{1}}, \bar{a}_{s}\right] \equiv\left[c_{t_{1}}, \bar{b}_{s}\right] \\
M \models \neg\left(\varphi\left[c_{t_{2}}, \bar{a}_{3}\right] \equiv \varphi\left[c_{t_{2}}, \bar{b}_{s}\right]\right) .
\end{gathered}
$$

Hence

$$
\varphi\left(x, \bar{a}_{s}\right) \in p\left(c_{t_{1}}, A, M, \Delta\right) \text { if and only if } \varphi\left(x, \bar{b}_{s}\right) \in p\left(c_{t_{1}}, A, M, \Delta\right)
$$

and

$$
\varphi\left(x, \bar{a}_{s}\right) \in p\left(c_{t_{2}}, A, M, \Delta\right) \text { if and only if } \varphi\left(x, \bar{b}_{s}\right) \notin p\left(c_{t_{2}}, A, M, \Delta\right) .
$$

So $p\left(c_{t_{1}}, A, M, \Delta\right) \neq p\left(c_{t_{2}}, A, M, \Delta\right)$, and as noted before this implies $T$ 
is $(\Delta, \chi)$-unstable, for every $\chi$.

Similarly we can prove

THEOREM 2.4. (1) If $T, \Delta \subset L_{\lambda^{+}, \omega} ;|T|+|\Delta| \leqq \lambda$, and for every $\kappa<\mu(\lambda), \kappa \in O d_{\Delta}(T)$, then every $\kappa \in O d_{\Delta}(T)$.

(2) If every $\kappa \in O d_{\Delta}(T)$, then $T$ is $\bar{\Delta}$-unstable.

REMARK. In 2.4.2 we use the following fact: if $M$ is $(\bar{\Delta}, \lambda)$-stable, $A \subset|M|,|A| \leqq \lambda, m<\omega$ then $\left|S^{m}(A, M, \Delta)\right| \leqq \lambda$.

THEOREM 2.5. Suppose $T \subset L_{\lambda^{+}, \omega},|T| \leqq \lambda,|L| \leqq \lambda$, and $T$ is unstable. Then there exists $\Delta_{1} \subset L_{\lambda^{+}, \omega},\left|\Delta_{1}\right| \leqq \lambda$ such that $T$ is $\Delta_{1}$-unstable.

Proof. As in the proof of Theorem 2.3, we depend on the method of Morley [10], Chang [1]. So let $T$ be $\Delta$-unstable. Without loss of generality, let $\Delta=\bar{\Delta}$ and $\Delta \subset L_{\kappa}{ }^{+}, \omega$. From Theorem 2.1 it follows that every $\mu \in O d_{\Delta}(T)$ [as $T$ is $\left(\Delta, 2^{2(\mu+\kappa+|A|+|L|)}\right)$-unstable]. Let $\lambda^{1}=$ $\mu(\lambda+|T|+\kappa+|\Delta|+|L|)$. So $T$ has a model $M$ such that $\lambda^{1} \in \operatorname{Od}_{\Delta}(M)$. We expand now $M$ to $M^{1}$ in the following way:

(1) For every subformula $\varphi(\bar{x})$ of a formula from $T \cup \Delta$ (including the formulas form $\Delta$ themselves) we add to $M$ the relation $R_{\varphi}^{M^{1}}=\{\bar{a}: M \vDash \varphi[\bar{a}]\}$.

(2) $M^{1}$ has Skolem function for every first-order formula in its language.

Let $L^{1}=L\left(M^{1}\right)$ be the first-order language associated with $M^{1}$. Clearly $\left|L\left(M^{1}\right)\right| \leqq|L|+|T|+|\Delta|+\kappa+\lambda$. As $\lambda^{1} \in O d_{\Delta}(M)$, there are $\bar{a}^{k}, k<\lambda^{1}$ from $M^{1}$ and there is $\varphi_{0}(\bar{x}, \bar{y}) \in \Delta$ such that $M^{1} \vDash \varphi_{0}\left[\bar{a}^{k}, \bar{a}^{l}\right]$ if and only if $k<l$. For simplicity we shall assume the sequences $\bar{a}^{k}$ are of length one, and $\bar{a}^{k}=\left\langle a_{k}\right\rangle$.

Hence there is a model $N$ and $a_{s} \in|N|$ for $s \in I$, which satisfy the following properties:

(1) the first-order language associated with $N$ is $L^{1}$.

(2) $N, M^{1}$ are elementarily equivalent.

(3) $N$ is a model of $T$, and for every subformula $\varphi(\bar{x})$ of a formula from $T \cup \Delta, N \models(\forall \bar{x})\left[\varphi(\bar{x}) \equiv R_{\varphi}(\bar{x})\right]$.

(4) I is an ordered set isomorphic to the rationals (s, $t$ will denote elements of $I$ ).

(5) for each $s, t \in I ; N \vDash \varphi_{0}\left[a_{s}, a_{t}\right]$ if and only if $s<t$.

(6) for each $c \in N$, there are $s_{1}<\cdots<s_{n}(\in I)$ and a term $B$ of $L^{1}$ such that

$$
N \vDash c=B\left[a_{s_{1}}, \cdots, a_{s_{n}}\right] .
$$

(7) for every $\varphi\left(x_{1}, \cdots, x_{n}\right) \in L^{1}, s_{1}<\cdots<s_{n}$, and $t_{1}<\cdots<t_{n}$ 
the following holds:

$$
N \vDash \varphi\left[a_{t_{1}}, \cdots, a_{t n}\right] \text { if and only if } N \vDash \varphi\left[a_{s_{1}}, \cdots a_{s_{n}}\right] .
$$

As $I$ is dense, by [7], [17], this holds also for every $\varphi \in L_{\infty, \omega}^{1}$.

Let $\bar{x}^{0}=\left\langle x_{0}, x_{1}\right\rangle, \bar{x}^{1}=\left\langle x_{2}, x_{3}\right\rangle$.

Let $\left\{\varphi_{k, n}\left(\bar{x}^{0}, \bar{x}^{1}, y_{0}, \cdots y_{n-1}\right): n<\omega, k<|L|\right\}$ be the list of the atomic formulas of $L$. Let

$$
\begin{aligned}
& \Phi_{n}\left(\bar{x}^{0}, \bar{x}^{1}, y_{0}, \cdots, y_{n-1}, z_{0}, \cdots, z_{n-1}\right)= \\
= & \bigwedge_{k<|L|}\left(\varphi_{k, n}\left(\bar{x}^{0}, \bar{x}^{1}, y_{0}, \cdots, y_{n-1}\right) \equiv \varphi_{k n}\left(\bar{x}^{0}, \bar{x}^{1}, z_{0}, \cdots, z_{n-1}\right)\right) \\
& \Phi\left(\bar{x}^{0}, \bar{x}^{1}\right)= \\
= & \left(\exists y_{0} \forall z_{0} \exists z_{1} \forall y_{1}, \exists y_{2} \forall z_{2} \exists z_{3} \forall y_{3}, \cdots, \exists y_{2 m} \forall z_{2 m} \exists z_{2 m+1} \forall y_{2 m+1}, \cdots\right)_{m<\omega} \\
& \quad\left[\neg \bigwedge_{n<\omega} \Phi_{n}\left(\bar{x}^{0}, \bar{x}^{1}, y_{0}, \cdots, y_{n-1}, z_{0}, \cdots, z_{n-1}\right)\right] \cdot
\end{aligned}
$$

By Shelah [14], for every $L$-model $M_{1}$, and $\bar{a}, \bar{b} \in\left|M_{1}\right|^{2}, M_{1} \vDash \Phi[\bar{a}, \bar{b}]$ if and only if $\bar{a}$ and $\bar{b}$ realizes different $L_{\infty, \omega}$-types (i.e., there is $\varphi\left(\bar{x}^{0}\right) \in L_{\infty, \omega}$ such that

$$
\left.M_{1} \vDash \varphi[\bar{a}], M_{1} \vDash \neg \varphi[\bar{b}]\right) .
$$

REMARK. The definition of the satisfaction of $\Phi[\bar{a}, \bar{b}]$ is selfevident. Discussion about languages with such expressions can be found in Keisler [6].

Hence we can find functions $F_{1}, \cdots, F_{n}, \cdots$ whose domains and ranges are $|N|$, each with a finite number of places such that:

(*) if $N_{1}$ is a submodel of a reduct of $N$, whose associated first order language include $L$, and $\left|N_{1}\right|$ is closed under the functions $\left\{F_{n}\right.$ : $n<\omega\}$ then for every $\bar{a}, \bar{b} \in\left|N_{1}\right|^{2}, N \models \Phi[\bar{a}, \bar{b}]$ implies $N_{1} \vDash \Phi[\bar{a}, \bar{b}]$.

Now as in the downward Lowenheim-Skolem theorem, we can find a model $N_{1}$ such that:

(A) $\left|N_{1}\right| \subset|N|,\left\{a_{s}: s \in I\right\} \subset\left|N_{1}\right|, \| N_{1}|| \leqq \lambda$ and $N_{1}$ is a submodel of a reduct of $N$.

(B) $\left|N_{1}\right|$ is closed under $\left\{F_{n}: n<\omega\right\}$

(C) if $\bar{a} \in\left|N_{1}\right|, \varphi(x, \bar{y})$ is a subformula of $\psi \in T$, and $N \models(\exists x) \varphi(x, \bar{a})$, then for some $b \in\left|N_{1}\right|, N \models \varphi[b, \bar{a}]$. Hence $N_{1}$ is a model of $T$.

(D) if $s_{1}<\cdots<s_{n}, t_{1}<\cdots<t_{n}, B$ is a term from $L^{1}$, and $B^{N}\left[a_{s_{1}}, \cdots, a_{s_{n}}\right] \in\left|N_{1}\right|$, then $B^{N}\left[a_{t_{1}}, \cdots, a_{t_{n}}\right] \in\left|N_{1}\right|$.

REMARK. Notice that by property (7) of $N$, if $B_{1}^{\mathrm{v}}\left[a_{s}, \cdots, a_{s_{n}}\right]=$ $B_{2}^{N}\left[a_{s_{1}}, \cdots, a_{s_{n}}\right]$ then $B_{1}^{N}\left[a_{t_{1}}, \cdots, a_{t_{n}}\right]=B_{2}^{N}\left[a_{t_{1}}, \cdots, a_{t_{n}}\right]$.

(E) The language of $N_{1}, L^{2}$, contains, $L$, is of cardinality $\lambda$, is contained in $L^{1}$, and for each $c \in\left|N_{1}\right|$ there is a term $B$ from $L^{2}$ such that $c=B^{N}\left[a_{s}, \cdots, a_{s_{n}}\right]$ for some $s_{1}<\cdots<s_{n}$. 
It is easy to prove that $N_{1}$ satisfies properties (6) and (7) of $N$, with $L^{1}$ replaced by $L^{2}$. It is also clear, by (C), that $N_{1}$ is a model of $T$. Let $s<t$, we know that $N \vDash \varphi_{0}\left[a_{s}, a_{t}\right]$, but $N \vDash \neg \varphi_{0}\left[a_{s}, a_{t}\right]$. Hence $\left\langle a_{s}, a_{t}\right\rangle,\left\langle a_{t}, a_{s}\right\rangle$ do that satisfy the same $L_{\infty \omega}$-type in $N$. By $(*)$ and $(\mathrm{B}),\left\langle a_{s}, a_{t}\right\rangle,\left\langle a_{t}, a_{s}\right\rangle$ also do not realize the same $L_{\infty \omega}$-type in $N_{1}$. As $\left\|N_{1}\right\| \leqq \lambda$, by Chang [1] it follows that $\left\langle a_{s}, a_{t}\right\rangle,\left\langle a_{t}, a_{s}\right\rangle$ do not

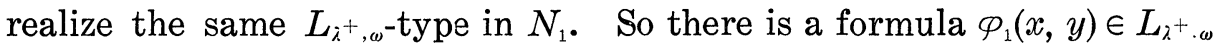
such that $N_{1} \vDash \varphi\left[a_{s}, a_{t}\right], N_{1} \vDash \neg \varphi\left[a_{t}, a_{s}\right]$. Let $\Delta_{0}=\left\{\varphi_{1}(x, y)\right\}, \Delta_{1}=\bar{\Delta}_{0}$. We shall prove that $T$ is $\Delta_{1}$-unstable, and so prove the theorem.

By Theorem 2.4.2 it suffices to prove that for every $\kappa, \kappa \in O d_{A_{1}}(T)$. Let $\kappa$ be any cardinal, and $J$ a dense order set, $I \subset J$, and $J$ contain a subset with order-type $\kappa$. We shall define now $N_{2}$ as an extension of $N_{1}$ such that:

$(\alpha) \quad\left\{a_{s}: s \in J\right\} \subset\left|N_{2}\right|$

( $\beta$ ) for every element $c$ of $N_{2}$ there are $s_{1}<\cdots s_{n} \in J$ and term $B \in L^{2}$ such that

$$
c=B^{N 2}\left[a_{s_{1}}, \cdots, a_{s_{n}}\right]
$$

$(\gamma)$ if $\varphi\left(x_{1}, \cdots, x_{n}\right)$ is an atomic formula, $s_{1}<\cdots<s_{n} \in J, t_{1}<\cdots<$ $t_{n} \in J$ then

$$
N_{2} \vDash \varphi\left[a_{s_{1}}, \cdots, a_{s_{n}}\right] \text { if and only if } N_{2} \vDash \varphi\left[a_{t_{1}}, \cdots, a_{t_{n}}\right] .
$$

It can be easily seen that $N_{2}$ exists. We can also show by in-

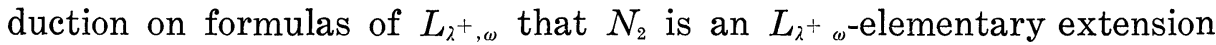
of $N_{1}$. (See [7], [17].) Hence $N_{2}$ is a model of $T$. It is also clear that for every $s, t \in J, N_{2} \vDash \varphi_{1}\left[a_{s}, a_{t}\right]$ if and only if $s<t$. By the definition of $J$ and $\Delta_{1}$ this implies $\kappa \in O d_{\Delta_{1}}\left(N_{2}\right)$ hence $\kappa \in O d_{\Delta_{1}}(T)$, and by 2.4.2, this implies $T$ is $\Delta_{1}$-unstable, where $\left|\Delta_{1}\right| \leqq \lambda,\left|\Delta_{1}\right| \subset L_{\lambda^{+}, \omega}$.

THEOREM 2.6. If $T$ is unstable, $T \subset L_{\lambda^{+} \omega}, \mu>\lambda+|T|$, then $T$ has exactly $2^{\mu}$ non-isomorphic models of cardinality $\mu$. (For most cases it suffices to demand $\mu \geqq \lambda+|T|+\aleph_{1}$.)

Proof. By Theorem 2.5, and Shelah [16].

\section{REFERENCES}

1. C. C. Chang, Some remarks on the model theory of infinitary languages, Lecture Notes in Math. No. 72, The syntax and semantics of infinitary languages, SpringerVerlag, Berlin, Heidelberg, New York, 1968, pp. 36-64.

2. A. Ehrenfeucht and A. Mostowski, Models of axiomatic theories admitting automorphisms, Fundamenta Math., 43 (1956), 50-68.

3. P. Erdös and A. Hajnal, Unsolved problems in set theory, Proc. of Symp. in Pure Math. XIII Part I A.M.S. Providence, R. I., (1971), 17-48.

4. P. Erdös, A. Hajnal and R. Rado, Partition relations for cardinal numbers, Acta 
Math., 16 (1965), 93-196.

5. P. Erdös and M. Makkai, Some remarks on set theory $X$, Studia Scientiarum Math. Hungarica, 1 (1966), 157-159.

6. H. J. Keisler, Formulas with linearly ordered quantifiers, Lecture Notes in Math. No. 72, The syntax and semantics of infinitary languages, Springer-Verlag, Berlin, Heidelberg, New York, (1968), 96-130.

7. M. Makkai, Structures elementarily equivalent to models of higher power relative to infinitary languages, Notices of Amer. Math. Soc., 15 (1969), 322.

8. W. Mitchell, On the cardinality of dense subsets of linear ordering II, Notices of Amer. Math. Soc., 15 (1968), 935.

9. M. Morley, Categoricity in power, Trans. Amer. Math. Soc., 114 (1965), 514-538.

10. - Omitting classes of elements, The theory of models, edited by J. W. Addison, L. Henkin and A. Tarski, Proceedings of the 1964 Intern. Symp. for Logic, Berkeley (Amsterdam, North-Holland Publ. Co.), (1965), 265-274.

11. F. P. Ramsey. On a problem of formal logic, Proceedings of the London Math. Society, Ser. 2, 30 (1929), 328-384.

12. S. Shelah, Stable theories, Israel J. Math., 7 (1969), 187-202.

13. - Finite diagrams stable in power, Annals of Math. Logic, 2 (1790), 69-116.

14. - On the number of non-almost isomorphic models, Pacific J. Math., 36 (1971), 811-818.

15. S. Shelah, Stability and the f.c.p.; Model theoretic properties of formulas in firstorder theories, Annals of Math. Logic, 3 (1971), 271-362.

16. - On the number of non-isomorphic models of an unstable first-order theory, Israel J. Math., 9 (1971), 473-487.

17. P. C. Eklof, On the existence of $L_{\infty, \kappa}$-indiscernible, Proc. Amer. Math. Soc., 25 (1970), 798-800.

18. P. Erdös and A. Hajnal, Unsolved and solved problems in set theory, to appear (in the Proc. of Tarski Symp.?)

19. M. Sauer, On the density of families of sets, J. Combinatorial Theory, Series A, 13, No. 1 (July 1972).

Received August 13, 1970. The preparation of this paper was supported in part by NSF Grant \# GP-22937.

The Hebrew University

PRINCETON UNIVERSITY

AND

University of California, Los ANGEles 



\section{PACIFIC JOURNAL OF MATHEMATICS}

\section{EDITORS}

H. SAMELSON

Stanford University

Stanford, California 94305

C. R. Hовву

University of Washington

Seattle, Washington 98105
J. DugundJI

Department of Mathematics

University of Southern California

Los Angeles, California 90007

RICHARD ARENS

University of California

Los Angeles, California 90024

\section{ASSOCIATE EDITORS}
E. F. BECKENBACH
B. H. NeumanN
F. WOLF
K. YOSHIDA

\section{SUPPORTING INSTITUTIONS}

UNIVERSITY OF BRITISH COLUMBIA

CALIFORNIA INSTITUTE OF TECHNOLOGY

UNIVERSITY OF CALIFORNIA

MONTANA STATE UNIVERSITY

UNIVERSITY OF NEVADA

NEW MEXICO STATE UNIVERSITY

OREGON STATE UNIVERSITY

UNIVERSITY OF OREGON

OSAKA UNIVERSITY
UNIVERSITY OF SOUTHERN CALIFORNIA

STANFORD UNIVERSITY

UNIVERSITY OF TOKYO

UNIVERSITY OF UTAH

WASHINGTON STATE UNIVERSITY

UNIVERSITY OF WASHINGTON

$\stackrel{*}{*} \stackrel{*}{*} \stackrel{*}{*}{ }^{*}$ AMERICAN MATHEMATICAL SOCIETY

NAVAL WEAPONS CENTER 


\section{Pacific Journal of Mathematics}

\section{Vol. 41, No. $1 \quad$ November, 1972}

Anatole Beck and Peter Warren, Weak orthogonality.................

Jonnie Bee Bednar and Howard E. Lacey, Concerning Banach spaces whose duals are abstract $L$-spaces.............................

Louis Harvey Blake, Canonical extensions of measures and the extension of regularity of conditional probabilities..........................

R. A. Brooks, Conditional expectations associated with stochastic processes ..........................................

Theodore Allen Burton and Ronald Calvin Grimmer, On the asymptotic behavior of solutions of $x^{\prime \prime}+a(t) f(x)=e(t) \ldots \ldots \ldots \ldots \ldots \ldots$

Stephen LaVern Campbell, Operator-valued inner functions analytic on the closed disc ............................................

Yuen-Kwok Chan, A constructive study of measure theory...

Alexander Munro Davie and Bernt Karsten Oksendal, Peak interpolation sets for some algebras of analytic functions ...................

H. P. Dikshit, Absolute total-effective $\left(N, p_{n}\right)(c, 1)$ method ...............

Robert E. Edwards, Edwin Hewitt and Kenneth Allen Ross, Lacunarity for

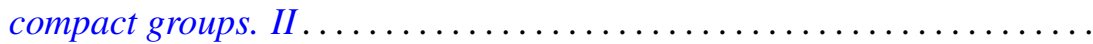

James Daniel Halpern, On a question of Tarski and a maximal theorem of Kurepa

Gerald L. Itzkowitz, A characterization of a class of uniform spaces that admit an invariant integral

Mo Tak Kiang, Semigroups with diminishing orbital diameters ..

Glenn Richard Luecke, A class of operators on Hilbert space ...

R. James Milgram, Group representations and the Adams spectral sequence. . .

G. S. Monk, On the endomorphism ring of an abelian p-group, and of a large subgroup...

Yasutoshi Nomura, Homology of a group extension ...

R. Michael Range, Approximation to bounded holomorphic functions on strictly pseudoconvex domains...

Norman R. Reilly, Inverse semigroups of partial transformations and $\theta$-classes.

Chris Rorres, Strong concentration of the spectra of self-adjoint operators

Saharon Shelah, A combinatorial problem; stability and order for models and theories in infinitary languages.

George Gustave Weill, Vector space decompositions and the abstract

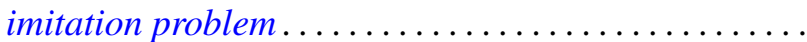

